\title{
MEDIAL IDEMPOTENT GROUPOIDS III
}

\author{
JUNG R. CHO and JÓZEF DUDEK
}

(Received 7 October 1998; revised 6 September 1999)

\author{
Communicated by B. A. Davey
}

\begin{abstract}
As a sequel to the previous two papers of the second author, we investigate the structure of medial idempotent groupoids by $p_{n}$-sequences. To complete the series of research, this paper has three purposes. First, we summarize some results in the previous papers so that this paper can cover the materials presented there. Secondly, using earlier results, we prove a few theorems which show the importance of the medial law in controlling the growth of $p_{n}$-sequences of groupoids. Finally, we state some problems and conjectures raised during the series of research.
\end{abstract}

2000 Mathematics subject classification: primary 08A40, $20 \mathrm{~N} 02$.

\section{Introduction}

In the class of groupoids, the idempotent law and the medial law have received a special attention because they appear very naturally in most branches of mathematics, and many authors have studied medial idempotent groupoids with many different approaches, sometimes under different names [26]. Especially, the medial law has received about ten different names in many different areas [1], and much research has been done on this identity by Ježek and Kepka in their monograph [19].

In this paper, as in the earlier papers of this series [10, 12], we approach medial idempotent groupoids by means of the number of essential term functions. Characterizing the structure of a groupoid or any algebra in general by its $p_{n}$-sequence has been an active research problem for many years, and it turned out that the $p_{n}$-sequence gives much information about an algebra, especially when the algebra is idempotent.

The first author was supported by the academic research fund of Ministry of Education, Republic of Korea, Project No. BSRI-97-1433.

(C) 2000 Australian Mathematical Society $0263-6115 / 2000 \$ A 2.00+0.00$ 
It is also true that the $p_{n}$-sequence is very hard to determine without the idempotent law. Many results on $p_{n}$-sequences of algebras are collected in the valuable survey article of Grätzer and Kisielewicz [17] with a detailed history, references and open problems. It is shown in [1] that the variety of medial idempotent groupoids and the variety of commutative idempotent groupoids are generated by affine spaces over prime fields, and so it is not surprising that affine spaces play a very important role in characterizing those groupoids in this way.

For an algebra $\mathbf{A}$, we denote by $p_{n}(\mathbf{A})$ the number of essentially $n$-ary term functions (or simply 'terms') of $\mathbf{A}$ for all $n \geq 1$. Note that $p_{0}(\mathbf{A})$ is the number of all constant unary terms of $\mathbf{A}$. The sequence $\left(p_{0}(\mathbf{A}), p_{1}(\mathbf{A}), p_{2}(\mathbf{A}), \ldots\right)$ is called the $p_{n}$-sequence of the algebra $\mathbf{A}$. Two algebras with the same set of term functions are said to be term equivalent and it is clear that term equivalent algebras have the same $p_{n}$-sequence. In this paper, we will treat term equivalent algebras as the same algebras.

A groupoid is called proper if the fundamental operation is essentially binary. A groupoid is called an n-polynomial groupoid if $p_{n}(G, \cdot)=n$ for all $n$, and these groupoids are completely characterized in [24].

For further concepts and terminology not defined in this paper, we refer the readers to $[10,16]$, especially for the definition of a Ptonka sum of algebras. We will say a Plonka sum of algebras is proper if the semilattice ordered system for the sum is not trivial and the algebras involved in the sum are not all trivial.

For simplicity, we use $x y^{n}$ for the term $(\cdots((x y) y) \cdots) y$ with $y$ repeated $n$ times, and use $x_{1} x_{2} \cdots x_{n}$ for $\left(\cdots\left(\left(x_{1} x_{2}\right) x_{3}\right) \cdots\right) x_{n}$. We also use the following notations.

$V_{\mathrm{i}}$ : the variety of all idempotent groupoids,

$V_{\mathrm{ci}}$ : the variety of all commutative idempotent groupoids,

$V_{\mathrm{mi}}$ : the variety of all medial idempotent groupoids,

$V_{\text {mci }}$ : the variety of all medial commutative idempotent groupoids,

SL: the variety of all semilattices,

DSQ : the variety of all distributive Steiner quasigroups,

NG : the class of all $n$-polynomial groupoids,

$\mathrm{RB}$ : the class of all proper rectangular bands,

$\operatorname{Aff}(q)$ : the class of all nontrivial affine spaces over $\mathrm{GF}(q)$,

$\operatorname{PA}(q)$ : the class of all proper Płonka sums of affine spaces over $\operatorname{GF}(q)$, where $\operatorname{GF}(q)$ is the Galois field with $q$ elements.

Affine spaces are introduced in [22], and it is proved in [25] that, for a prime number $p$, if $(A, F) \in \operatorname{Aff}(p)$ then $(A, F)$ is term equivalent to the algebra $(A, f)$ for any essentially $n$-ary operation $f$ in $F$ with $n \geq 2$. Thus, we regard $\operatorname{Aff}(p)$ as the class of groupoids $(G,((p+1) / 2)(x+y))$, where $(G,+)$ is an Abelian group of exponent $p$ (see also [10]). Then it is clear to see that $\operatorname{Aff}(p) \subseteq V_{\text {mci. }}$. By [23], it also follows that $\operatorname{PA}(p) \subseteq V_{\text {mci. }}$ 


\section{Some characterization of affine spaces}

In this section, we summarize the main results of the previous two papers of this series of research and some other results related to them so that this paper can be used as a reference in the future.

The following are the main results of the previous two papers of this series of research.

THEOREM $2.1([10,12])$. Let $(G, \cdot)$ be a nontrivial groupoid in $V_{\mathrm{mi}}$. Then,

(i) $p_{2}(G, \cdot)=0$ if and only if $(G, \cdot)$ satisfies $x y=x$ or $x y=y$;

(ii) $p_{2}(G, \cdot)=1$ if and only if $(G, \cdot) \in \mathrm{SL} \cup \operatorname{Aff}(3)$;

(iii) $p_{2}(G, \cdot)=2$ if and only if $(G, \cdot) \in \mathrm{NG} \cup \mathrm{RB} \cup \operatorname{Aff}(4)$;

(iv) $p_{2}(G, \cdot)=3$ if and only if $(G, \cdot) \in \operatorname{Aff}(5) \cup \operatorname{PA}(3)$;

(v) $p_{2}(G, \cdot)=5$ if and only if $(G, \cdot) \in \operatorname{Aff}(7) \cup \operatorname{PA}(5)$.

In the proof of the Theorem 2.1, the following result was critical.

THEOREM $2.2([10])$. If $(G, \cdot) \in V_{\mathrm{mi}}$ and $p_{3}(G, \cdot)=3$ then $(G, \cdot)$ is term equivalent to a groupoid in $V_{\mathrm{mci}}$.

The next two theorems give more results related to Theorem 2.1 and explain some results of the theorem in more detail.

THEOREM 2.3. Let $(G, \cdot)$ be a nontrivial groupoid in $V_{\text {mci. }}$. Then,

(i) $(G, \cdot) \in \mathrm{SL}$ if and only if either $x y^{2} \in\left\{x y, y x^{2}, x y^{3}\right\}$ or $x y^{2} x \in\left\{y x^{2} y, y x^{2}\right.$, $\left.x y^{3}, y x^{3}\right\}([6,12])$;

(ii) $(G, \cdot) \in \operatorname{Aff}(3)$ if and only if $x y^{2} x=x$ (equivalently $\left.x y^{2}=x\right)([10,18])$;

(iii) $(G, \cdot) \in \operatorname{Aff}(5)$ if and only if $x y^{2} x=y([10,18])$;

(iv) $(G, \cdot) \in \operatorname{Aff}(5)$ if and only if $x y^{3}=y x^{2}$ and $p_{2}(G, \cdot)=3([10])$;

(v) $(G, \cdot) \in \operatorname{Aff}(7)$ if and only if it $x y^{3}=x([12])$;

(vi) $(G, \cdot) \in \mathrm{PA}(3)$ if and only if $(G, \cdot) \notin \mathrm{SL}$ and either $x y^{2} x=x y^{2}, x y^{3}=y x^{3}$ or $x y^{3}=x y([12])$;

(vii) $(G, \cdot)$ is an affine module over $\mathbb{Z}_{d}$ with $d \mid 2^{n}-1$ if and only if $x y^{n}=x([4])$;

(viii) $(G, \cdot)$ is a Plonka sum of affine modules over $\mathbb{Z}_{d}$ with $d \mid 2^{n}-1$ if and only if $x y^{n+1}=x y([6])$.

THEOREM $2.4([10,12])$. Let $(G, \cdot)$ be a nontrivial groupoid in $V_{\mathrm{mi}}$. Then,

(i) if $x y^{3}=y x^{2}$, then $(G, \cdot) \in \operatorname{Aff}(5)$ if and only if $p_{2}(G, \cdot)=3$;

(ii) if $x y^{2}=x$ or $x y^{2}=y x^{2}$, then $(G, \cdot) \in \operatorname{Aff}(7)$ if and only if $p_{2}(G, \cdot)=5$;

(iii) if $x y^{3}=x y$, then $(G, \cdot) \in \operatorname{PA}(3)$ if and only if $p_{2}(G, \cdot)=3$;

(iv) if $x y^{2}=y x$, then $(G, \cdot) \in \operatorname{PA}(5)$ if and only if $p_{2}(G, \cdot)=5$. 


\section{3. $p_{n}$-sequences and the medial law}

In this section, we present a few theorems which show how easily the medial law is implied even by a relatively loose restriction on the growth of $p_{n}$-sequences. First, we recall some known results for the later use.

LeMMA 3.1. (i) Let $(G, \cdot) \in V_{\mathrm{i}}$. Then $p_{2}(G, \cdot)<6$ if and only if $(G, \cdot) \in$ $S L \cup D S Q \cup R B \cup N G([7])$.

(ii) If $(G, \cdot) \in V_{\text {ci }}$ and $(G, \cdot) \notin \operatorname{Aff}(3) \cup \mathrm{SL}$, then $p_{n}(G, \cdot) \geq 3^{n-1}$ for all $n \geq 4$ ([8]).

(iii) $\mathrm{NG} \subseteq V_{\mathrm{mi}}([13])$.

(iv) If $(G, \cdot) \in V_{\mathrm{i}}$ and $(G, \cdot) \notin \operatorname{Aff}(2) \cup \operatorname{SL} \cup \mathrm{RB}$, then $p_{n+1}(G, \cdot) \geq p_{n}(G, \cdot)+1$ for all $n \geq 2$ ([20]).

THEOREM 3.1. If $(G, \cdot)$ is a nontrivial groupoid in $V_{\mathrm{i}}$ such that $p_{n}(G, \cdot) \leq n$ for some $n \geq 4$, then $(G, \cdot)$ is medial.

Proof. Suppose that $p_{n}(G, \cdot) \leq n$ for some $n \geq 4$. We claim that $p_{3}(G, \cdot)<6$. Assume $p_{3}(G, \cdot) \geq 6$. Then $(G, \cdot) \notin \operatorname{SL} \cup \operatorname{RB} \cup \operatorname{Aff}(2)$, since $p_{3}(G, \cdot) \leq 2$ otherwise. Thus, by Lemma 3.1(iv), we have that $p_{n+1}(G, \cdot) \geq p_{n}(G, \cdot)+1$ for all $n \geq 2$. Then, for all $n \geq 3$, we have that $p_{n}(G, \cdot) \geq p_{n-1}(G, \cdot)+1 \geq p_{n-2}(G, \cdot)+2 \geq$ $\cdots \geq p_{3}(G, \cdot)+(n-3) \geq 6+(n-3)=n+3$, which is a contradiction to our hypothesis. Thus, $p_{3}(G, \cdot)<6$ as we claimed. Now, by Lemma 3.1(i), $(G, \cdot) \notin$ $\mathrm{SL} \cup \mathrm{RB} \cup \mathrm{NG} \cup \mathrm{DSQ}$. Obviously, $(G, \cdot)$ is medial if $(G, \cdot) \in \mathrm{SL} \cup \mathrm{RB}$. By Lemma 3.1(iii), $(G, \cdot)$ is medial if $(G, \cdot) \in$ NG. Now suppose $(G, \cdot) \in$ DSQ and $(G, \cdot) \notin$ SL. Then $(G, \cdot) \in \operatorname{Aff}(3)$ by Lemma 3.1(ii) and so $(G, \cdot)$ is medial.

REMARK 3.1. We note that the bound $n \geq 4$ in the above theorem is the best possible. In fact, a distributive Steiner quasigroup has only three ternary terms, namely $(x y) z,(y z) x$ and $(z x) y$, but there exist nonmedial distributive Steiner quasigroups [27].

Following [15], we say that a sequence $\mathbf{a}=\left(a_{0}, a_{1}, a_{2}, \ldots\right)$ has a subexponential growth if there exists a positive constant $c$ such that $a_{n} \leq c^{n}$ for all $n$, and that a has a subpolynomial growth if there exists a polynomial $\varphi(x)$ in $\mathbb{Z}[x]$ such that $a_{n} \leq \varphi(n)$ for all $n$.

An identity is called regular if both sides of the identity involve the same variables.

THEOREM 3.2. If $(G, \cdot) \in V_{\mathrm{ci}}$ and the $p_{n}$-sequence of $(G, \cdot)$ has a subexponential growth, then $(G, \cdot)$ is medial. 
PROOF. Assume that $(G, \cdot) \in V_{\mathrm{ci}}$ and there is a constant $c$ such that $p_{n}(G, \cdot) \leq c^{n}$ for all $n$. Using [15, Theorem 3], we can deduce that $(G, \cdot)$ is a semilattice, an affine space over $\mathbb{Z}_{d}$ or a Płonka sum of affine spaces over $\mathbb{Z}_{d}$ for some odd integer $d$. A semilattice is obviously medial. An affine space over $\mathbb{Z}_{d}$ is term equivalent to a groupoid $(G,((d+1) / 2)(x+y))$ for some Abelian group $(G,+)$ of exponent $d$ (see [6]), which is certainly medial. Note that a Plonka sum of groupoids preserves regular identities common to all groupoids involved in the sum (see [23]). Since affine spaces are medial and the medial law is a regular identity, Plonka sums of affine modules are also medial.

THEOREM 3.3. If $(G, \cdot) \in V_{\mathrm{ci}}$ and the $p_{n}$-sequence of $(G, \cdot)$ has a subpolynomial growth, then $(G, \cdot)$ is a semilattice (and hence medial).

PROOF. Assume that $(G, \cdot) \in V_{\mathrm{ci}}$ and $p_{n}(G, \cdot) \leq \varphi(n)$ for some polynomial $\varphi(x)$ in $\mathbb{Z}[x]$ and for all $n$. Then there exist a positive integer $k$ and a constant $c$ such that $p_{n}(G, \cdot)<c n^{k}$ for all $n$. Then, by [15, Theorem 2], $(G, \cdot)$ is a semilattice and hence medial.

REMARK 3.2. Theorem 3.3 is not true in general if the groupoid is not commutative. In fact, we find in [3] noncommutative idempotent nonmedial groupoids $(G, \cdot)$ with $p_{n}(G, \cdot)=n^{2}$ for all $n$.

Now we introduce some earlier results which are useful to prove the next theorem.

LEMMA 3.2. (i) If $(G, \cdot) \in V_{\mathrm{ci}}$ then $x y^{n} \neq y$ in $(G, \cdot)$ for all $n([5])$.

(ii) If $(G, \cdot) \in V_{\mathrm{i}}$, then $(G, \cdot) \in \mathrm{SL}$ if and only if $(G, \cdot)$ is a distributive commutative groupoid satisfying $x y^{2}=y x^{2}([6])$.

(iii) If $(G, \cdot) \in V_{\mathrm{mci}}, x y^{2}$ is essentially binary and $x y^{2} \neq y x^{2}$, then $p_{n}(G, \cdot) \geq 3^{n-1}$ for all $n \geq 1([8])$.

THEOREM 3.4. If $(G, \cdot) \in V_{\mathrm{ci}}$ and $p_{2}(G, \cdot)>1$, then $x y^{2}$ is essentially binary and $p_{n}(G, \cdot) \geq 3^{n-1}$ for all $n \geq 4$. If, in addition, $(G, \cdot)$ is medial, then $x y^{2} \neq y x^{2}$ and $p_{n}(G, \cdot) \geq 3^{n-1}$ for all $n \geq 1$.

Proof. Suppose $(G, \cdot) \in V_{\text {ci }}$ and $p_{2}(G, \cdot)>1$. Since $p_{2}(G, \cdot)>1$, we infer that $(G, \cdot)$ is not a semilattice and $(G, \cdot) \notin \operatorname{Aff}(3)$ by Theorem 2.1. Then, by Lemma 3.1(iii), we obtain that $p_{n}(G, \cdot) \geq 3^{n-1}$ for all $n \geq 4$. If $x y^{2}=x$ then $(G, \cdot)$ is a Steiner quasigroup and so $p_{2}(G, \cdot) \leq 1$, a contradiction. By Lemma 3.2(i), we have $x y^{k} \neq y$ for all $k$, and hence $x y^{2} \neq y$. Thus $x y^{2}$ is essentially binary. Suppose, furthermore, that $(G, \cdot)$ is medial. Then $(G, \cdot)$ is distributive by the medial law and the idempotent law. If $x y^{2}=y x^{2}$ then $(G, \cdot) \in$ SL by Lemma 3.2(ii) and so 
$p_{2}(G, \cdot)=1$, a contradiction. Hence $x y^{2} \neq y x^{2}$. Then, by Lemma 3.2(iii), we obtain that $p_{n}(G, \cdot) \geq 3^{n-1}$ for all $n \geq 1$, which completes the proof.

\section{The medial law and minimal clones}

A set of term functions of an algebra is called a clone of the algebra if it contains all projections and is closed under taking compositions. A clone is called minimal if it properly contains only the trivial clone, that is, the clone consisting of projections only.

LeMma 4.1. Let $(G, \cdot) \in V_{\mathrm{ci}}$ satisfy $x y^{n}=x$ for some $n \geq 2$. Then the clone of $(G, \cdot)$ is minimal if and only if $(G, \cdot) \in \operatorname{Aff}(p)$ for some prime $p$ dividing $2^{n}-1$ (compare with Theorem 2.3(vii)).

THEOREM 4.1. Let $(G, \cdot) \in V_{\text {ci }}$.

(i) If $(G, \cdot)$ satisfies $x y^{n}=x$ for some $n \geq 1$ and the clone of $(G, \cdot)$ is minimal then $(G, \cdot)$ is medial.

(ii) If $(G, \cdot)$ is a cancellative groupoid and $p_{2}(G, \cdot)$ is finite, then the clone of $(G, \cdot)$ is minimal if and only if $(G, \cdot) \in \operatorname{Aff}(p)$ for some prime $p \geq 3$.

PROOF. (i) follows from the Lemma 4.1, since any affine space over $\mathrm{GF}(p)$ with $p \geq 3$ is term equivalent to the medial groupoid $(G,((p+1) / 2)(x+y))$ by Lemma 3.1, where $(G,+)$ is an Abelian group of exponent $p$. (ii) Since $p_{2}(G, \cdot)$ is finite, we see that the mapping $k \mapsto x y^{k}$ is not injective. Thus $x y^{i}=x y^{j}$ for some distinct integers $i$ and $j$, say $j \geq i$. Then, by the cancellativity, we have that $x y^{j-i}=x$ and the conclusion follows by Lemma 4.1 .

THEOREM 4.2. Let $\mathbf{A}$ be an algebra with $p_{2}(\mathbf{A})=3$. Then the following statements are equivalent.

(i) $\mathbf{A} \in \operatorname{Aff}(5)$.

(ii) The clone of $\mathbf{A}$ is minimal.

(iii) $\mathbf{A} \in V_{\text {mci }}$ and $\mathbf{A}$ satisfies $x y^{2} x=y$.

(iv) A is a groupoid with $p_{n}(\mathbf{A})=\left(4^{n}-(-1)^{n}\right) / 5$ for all $n$.

(v) $\mathbf{A} \in V_{\mathrm{mi}}$ and $\mathbf{A}$ satisfies a nonregular identity.

Proof. (i) is equivalent to (ii) by [9]. (i) is equivalent to (iii) by Theorem 2.3(iii). (i) is equivalent to (iv) by [2] and [11]. Clearly (iii) implies (v). It remains only to show (v) implies (i). By Theorem 2.1, A $\in$ PA(3) or $\mathbf{A} \in \mathbf{A f f}$ (5). Since a Plonka sum satisfies only regular identities (see [23]), we have the latter case. 
THEOREM 4.3. Let $\mathbf{A}$ be an algebra with $p_{2}(\mathbf{A})=5$. Then the following statements are equivalent.

(i) $\mathbf{A} \in \operatorname{Aff}(7)$.

(ii) The clone of $\mathbf{A}$ is minimal.

(iii) $\mathbf{A} \in V_{\mathrm{mci}}$ and $\mathbf{A}$ satisfies $x y^{3}=x$.

(iv) $\mathbf{A} \in V_{\mathrm{mi}}$ and $\mathbf{A}$ satisfies a nonregular identity.

ProOF. (i) is equivalent to (ii) by [14] and (i) is equivalent to (iii) by Theorem 2.3(v). Clearly (iii) implies (iv). We prove (iv) implies (i). By Theorem 2.1, $\mathbf{A} \in \mathrm{PA}(5)$ or $\mathbf{A} \in \mathbf{A f f}(7)$. Since a Płonka sum satisfies only regular identities, $\mathbf{A} \in \mathbf{A f f}(7)$.

\section{Problems and conjectures}

We present here some problems and conjectures.

PROBLEM 5.1. Characterize all medial idempotent groupoids $(G, \cdot)$ with $p_{2}(G, \cdot)=$ $2 d-1$ for some $d>1$. Is it true that if $p_{2}(G, \cdot)=2 d-1$, then $(G, \cdot)$ is a medial idempotent groupoid if and only if $(G, \cdot)$ is an affine module over $\mathbb{Z}_{2 d+1}$ or a proper Płonka sum of affine modules over $\mathbb{Z}_{2 d-1}$ (see Theorem 2.3 (vii) and (viii))? Note that the problem is solved in the positive for $d=2,3([10,12])$. In these cases, the affine modules in question are simply affine spaces.

Problem 5.2. Characterize medial idempotent groupoids $(G, \cdot)$ with $p_{2}(G, \cdot)=4$. Note that all medial idempotent groupoids with $p_{2}(G, \cdot) \in\{0,1,2,3,5\}$ were classified (Theorem 2.1).

PROBLEM 5.3. Characterize medial idempotent groupoids $(G, \cdot)$ satisfying a nonregular identity and $p_{2}(G, \cdot)=p$ for a prime number $p$. Are these groupoids affine spaces over $\mathrm{GF}(p)$ ? (See theorems in Section 4.)

CONJECTURE 5.4. For a prime number $p \geq 3$, the following statements are equivalent for a nontrivial groupoid $(G, \cdot)$.

(i) $(G, \cdot)$ is a nontrivial affine space over $\mathrm{GF}(p)$.

(ii) $p_{n}(G, \cdot)=\left((p-1)^{n}-(-1)^{n}\right) / p$ for all $n$.

(iii) The sequence $\left(0,1, p-2, p^{2}-3 p+3,(p-2)\left(p^{2}-2 p+2\right)\right)$ determines the $p_{n}$-sequence of $(G, \cdot)$ in the class of all groupoids.

In [14], it was conjectured that the statement (i) of Conjecture 5.4 is equivalent to the following.

(iv) The clone of $(G, \cdot)$ is minimal and $p_{2}(G, \cdot)=p-2$ for all $p \geq 5$. 
However, Lévai and Pálfy [21] showed that this conjecture is not true for prime numbers of the form $3 k+2$ with $k \geq 2$. Note that the equivalence of (i) and (iv) is true for $p=5$ and $p=7$. For $p=3$, the equivalence of all the above statements is true $[12,18]$.

\section{References}

[1] J. R. Cho, Varieties of medial algebras (Ph. D. Thesis, Emory University, Atlanta, 1986).

[2] B. Csákány, 'On affine spaces over prime fields', Acta Sci. Math. (Szeged) 37 (1975), 33-36.

[3] S. Crvenković and J. Dudek, 'Rectangular groupoids', Czechoslovak Math. J. 35 (1985), 405-414.

[4] J. Dudek, 'Medial groupoids and Mersenne's numbers', Fund. Math. 114 (1981), 109-112.

[5] - 'On binary polynomials in idempotent commutative groupoids', Fund. Math. 120 (1984), $187-191$.

[6] —_, 'Varieties of idempotent commutative groupoids', Fund. Math. 120 (1984), 193-204.

[7] — 'Polynomial characterization of some idempotent algebras', Acta Sci. Math. (Szeged) 50 (1986), 39-49.

[8] — , 'Polynomials in idempotent commutative groupoids', Dissertationes Math. 286 (1989), $1-55$

[9] _ , 'The unique minimal clone with three essentially binary operations', Algebra Universalis 27 (1990), 201-209.

[10] —-, 'Medial idempotent groupoids I', Czechoslovak Math. J. 41 (1991), 249-259.

[11] - 'On Csákány’s problem concerning affine spaces', Acta Sci. Math. (Szeged) 46 (1992), 3-13.

[12] - 'Medial idempotent groupoids II', in: Proceeding of the workshop on general algebra, Contribution to general algebras 9 (Linz, 1994), pp. 133-150.

[13] — ' 'On varieties of groupoid modes', Demonstratio Math. 27 (1994), 815-818.

[14] , 'Another unique minimal clone', preprint.

[15] J. Dudek and A. Kisielewicz, 'Idempotent algebras with log-linear free spectra', Algebra Universalis 28 (1991), 119-127.

[16] G. Grätzer, Universal algebra, 2nd edition, (Springer, New York, 1979).

[17] G. Grätzer and A. Kisielewicz, 'A survey of some open problems on $p_{n}$-sequences and free spectra of algebras and varieties', in: Universal algebra and quasigroup theory (eds. A. Romanowska and J. D. H. Smith), (Helderman, Berlin, 1992), pp. 57-88.

[18] G. Grätzer and R. Padmanabhan, 'On commutative, idempotent and nonassociative groupoids', Proc. Amer. Math. Soc. 28 (1971), 75-78.

[19] J. Ježek and T. Kepka, 'Medial groupoids', Rozpravy ČSAV, Řada Mat. Přírod. Věd 93 (1983).

[20] A. Kisielewicz, 'The $p_{n}$-sequences of idempotent algebras are strictly increasing II', Algebra Universalis 27 (1990), 261-269.

[21] L. Lévai and P. Pálfy, 'On binary minimal clones', Acta Cybernet. 12/3 (1996), 279-294.

[22] F. Ostermann and J. Schmidt, 'Der baryzentrische Kalkül als axiomatische Grundlage der affinen Geometrie', J. Reine Angew. Math. 224 (1966), 44-57.

[23] J. Płonka, 'On equational classes of abstract algebras defined by regular equations', Fund. Math. 64 (1969), 241-247.

[24] —, 'On algebras with $n$ distinct essentially $n$-ary operations', Algebra Universalis 1 (1971), 73-79.

[25] _ , 'R-prime idempotent reduct of abelian groups', Archiv. Math. (Basel) 24 (1973), 129-132. 
[26] A. Romanowska and J. D. H. Smith, Modal theory, an algebraic approach to order, geometry and convexity (Helderman, Berlin, 1985).

[27] J. Soublin, 'Étude algébrique de la notion de moyenne', J. Math. Pures Appl. 50 (1971), 53-264.

Department of Mathematics

Pusan National University

Pusan 609-735

Korea

e-mail: jungcho@hyowon.pusan.ac.kr
Mathematical Institute University of Wrocław pl. Grunwaldzki $2 / 4$ 50-384 Wrocław Poland e-mail: dudek@math.uni.wroc.pl 